\title{
A Multiscale Particle Filter and Multifeature- Based Contour Detection
}

\author{
Devshri Swami, Bahrti Chaurasia and Yashwant Kurmi
}

\begin{abstract}
In this paper, a multi-scale particle filter (MSPF) based algorithm and multi-feature-based surround inhibition (MFBSI) algorithm are proposed for contour detection in natural images. The contour detection algorithms are jointly tracks at two edgelets as edge elements. This multi-scale edgelet structure gathers the semi-local information as Bayesian modelling. The model is further approximate by using Monte Carlo algorithm. The individual surround inhibition weights of the feature as, including luminance, luminance contrast, and orientation. The scale-guided strategy is used for features together. The final surround inhibition is modulated using the combined weights in the neuron. The luminance contrast and luminance are proven as an excellent contour extraction capability. The multiscale particle filter (MSPF) based approach provides a better contour for the object segmentation.
\end{abstract}

Keywords

Classical receptive field,

Multiscale filtering,

Multiscale contour detection,

Statistical model,

Surround inhibition

\section{INTRODUCTION}

Contour detection is an essential task for processing image [1]. This process also essentially required for segmentation [2], recognition, tracking etc. [3]. Internal changes in natural imagery make this work an appropriate challenge. Here, describe an eye in a specific chain of apparently main, connected pixels. It is accompanied by the significance of the accompanying grouping and continuity characteristics for the visual realization of the human [4]. Unusual geometrical patterns are most difficult for contour detection [5]. Good continuity and arbitrator properties will serve as inspiration for the contour detector [6]. The procedure is a more general, but efficient method that integrates the comic and contours of the two regions. It considers the removal of contour with energy reduction [7]. It clearly encodes different contour cues by introduce
2D area labels and the 1D curve label. Then use the restrictions to ensure the consolidation of the contour label [8]. This first segment uses the field method to measure segmentation results by object and then contour [9]. The framework incorporates both the contour signals and regional signals in global optimization. In addition, the primary model is related to object shape. The edges are defined mainly in two different scales [10]. The algorithm may locally manage the calculation of each scale by ordering by the edges with the edges. The visual system based on cue combination and contextual modulation in multifeature-based surround inhibition (MFBSI) method [11]. The local features contribution is also considered in cluttered natural scenes for contour identification. For the selective surround self-consciousness weights are computed first, from classical receptive field (CRF), non-CRF contrast and orientation play important roles [12]. A scaleguided amalgamation approach is applied for combining the cues reticence weights under the proper control depending on statistical data relating CRF values at two separate ranges of data. The inhibition weights may modulate with the potency of surround reticence. Any multidimensional image has gradient value among the different channels based on this principle multiscale particle filter and multifeature-based contour detection method is proposed in this paper.

The paper is arranged as follows. Section II, introduction and analysis of the model and algorithm analysis. In Section III, result analysis followed by concluding remarks.

\section{Modelling AND AnAlytical Procedures}

The modelling of the multiscale particle filter approach is practically difficult process. The framework is proposed here is consisting of twoscales and intermediate dots connected dots or points. For an example a set of ? four points scale as Eq (1).

$$
E=\left(E^{1}, \ldots \ldots, E^{\square}\right) \in \digamma_{E} \subset \Omega_{E}^{\square}
$$

$E^{i}$ is consider as point which is consider in the image domain. The contour detection process is 
basically spatial tracking method [13]. The four local features are then used with the learning models.

Vector E brings together a small part of the concert, more information regarding the contour in comparison with a classic pixel-based source. By considering their previous value obtained from the distributions, it forces the mathematical limitations which can reduce the efficiency of an algorithm.

The offline learning gives the course features of the edges of the objects in the image. The initialization of edgelets is done in the previous section [14]. Scale-guided combination approach is used to combine the three inhibitory weights, which is then modulated by the surround inhibition. To indicate overall contours randomly, tracing algorithm is work satisfactory. The multi-scale edgelet obtained from previous result.

The transition distributions $p\left(E_{t} \vee E_{t-1}, e_{t-1}\right)$ and $p\left(e_{t} \vee E_{t}, e_{t-1}\right)$ are basic variance. These distributions are conditioned by $e_{t-1}$ is the basic information from previous experience.

A gradient norm matrix $\left|\Delta I_{e}\right|$ is basically $2 \times 2$. Is used in the gradient feature $f_{e}^{1}$ is totaled along the edgelets $e_{\tau}$ by Eq. (2)

$$
f_{e}^{1}\left(e_{t}, I_{e}\right)=\varnothing\left(\left(\nabla I_{e}\left(e_{t}^{i}\right) \mid\right)_{1 \leq i \leq M}\right)
$$

The texture features should have the perception regarding the pixel values for the initial segment different from the other.

\section{A. Orientation Gradient}

From the histogram gradients are calculated by using the same notations as in except, the histogram contains $\mathrm{R}=4 \mathrm{Rm}$ due to orientations (horizontal,vertical, and two-side diagonals) and Rm represents themagnitude bin number. All edgelet point $e_{i}^{t}$, information is stores into the histogram profile of its four point gradient values.

Gradient profile is learned and marked by a patent gradients profile model. In this process, it compares the printed profiles obtained from the image to the image. Gradient standard size is more reliable than normal gradient standard values in the presence of clusters.

\section{B. Multifeature Based Contour Detection}

The execution, extraction and combination based on local features based properties are performed with centre surround interaction for contour detection. The flowchart of the MFCD is shown in Fig. 1. It is a parallel processing of local features and the CRF weights.

The edge information transform in fine-scale consisting of more accurate localization, with more details for the specific point of interest. The isotropic non-specific is required to measure for the surround inhibition by using convolution method by using the Eq. (3),

$$
h(x, y)=€(x, y ; \sigma) * W d(x, y)
$$

here, $€(\mathrm{x}, \mathrm{y} ; \sigma)$, the CRF response map. The term $\mathrm{Wd}(\mathrm{x}, \mathrm{y})$ used here for the distance-based weighting function calculation. The final responses of the desire points of the contours are available by subtracting the resultant $\mathrm{W} \operatorname{com}(\mathrm{x}, \mathrm{y})$ by the Eq. (4) $r(x, y)=H(€(x, y ; \sigma)-\alpha \cdot W \operatorname{com}(x, y) \cdot h(x, y))(4)$

The $\alpha$, is the connection factor use to indicate the CRF strength related to surrounding non-CRF.

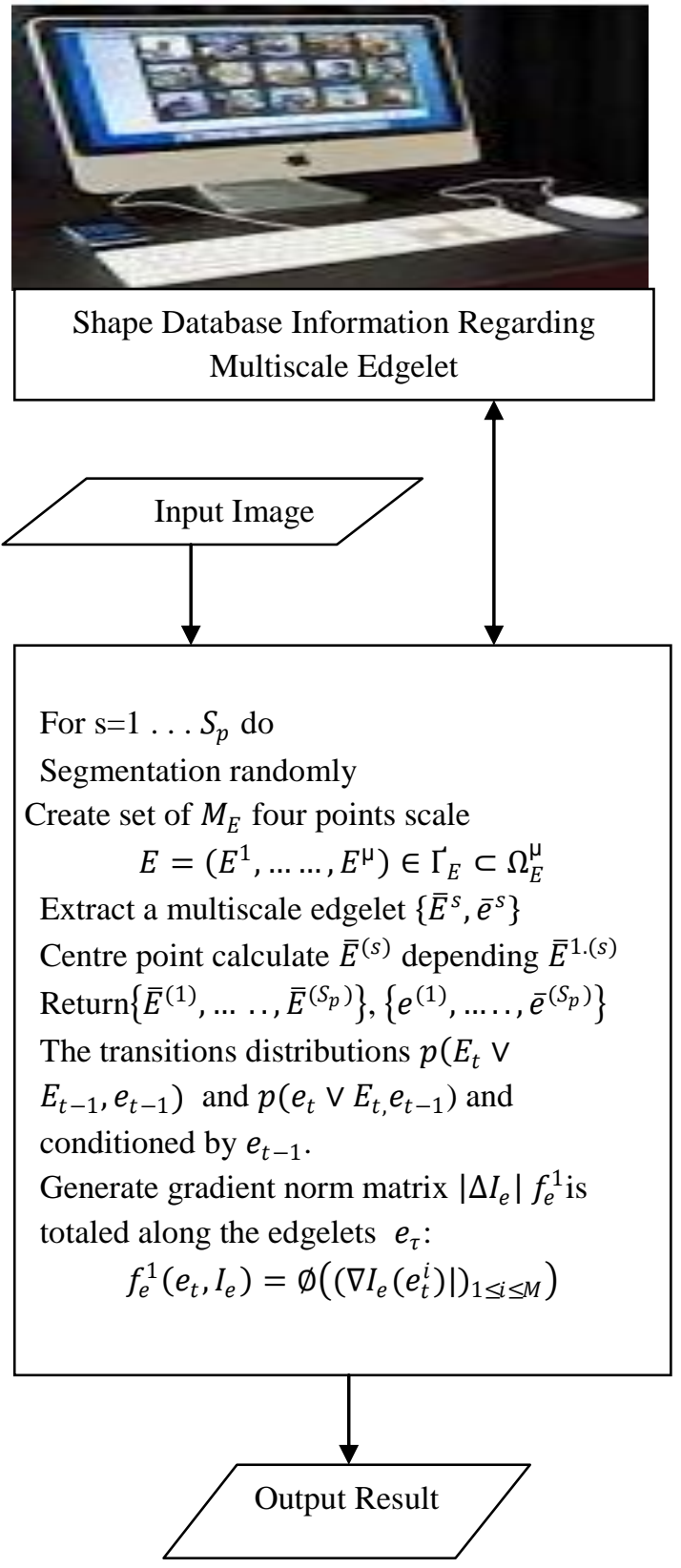

Fig.1. Algorithmic flow chart of the procedure.

Devshri Swami, Bharti Chaurasia and Yashwant Kurmi, "A Multiscale Particle Filter and Multi-Feature Based Contour Detection," International Journal of Advanced Engineering and Management, Vol. 2, No. 6, pp. 127-130, 2017. https://doi.org/10.24999/IJOAEM/02060031 


\section{RESUlt ANALYSIS}

The pre assumption or pre predicted irregular contour or user define contour the proposed method is able to obtain a correct contour path. First of all, all pixels that are not included in rough contours are considered banned. The intermediate control point area automatically spreads rough contour in the regular. By user's interaction, the rough contour path is achieved in ordered. The output of a traditional image and resultant output is shown in Fig. 2. The core of this structure is the design of the limitation with the following three features: First, the topological determination of limitations must be ensured. For an example, areas of different labels required to separate as per the requirement by contour and should not refuse by the target object borders. Secondly, these limitations should not be so vulnerable that there is no effective solution. Like jet stream algorithm, this particle filter-based technology is suitable for semi-automatic and / or one identification function. In contrast to predictive methods, this particle filtering methodology is automatic process and contextually dependent and it treat semi local region.

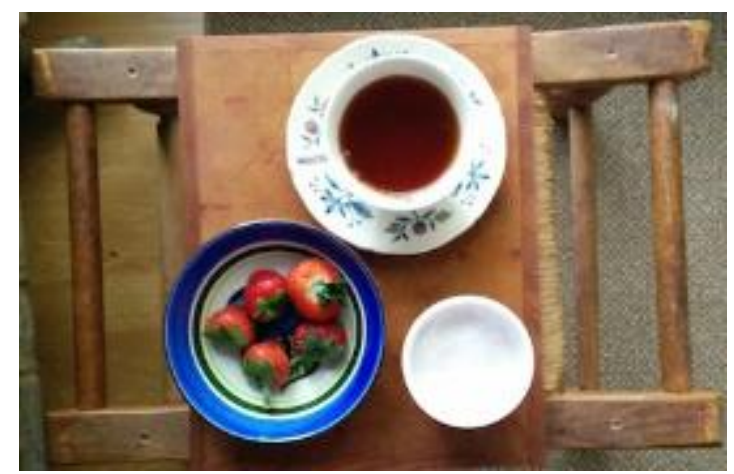

(a)

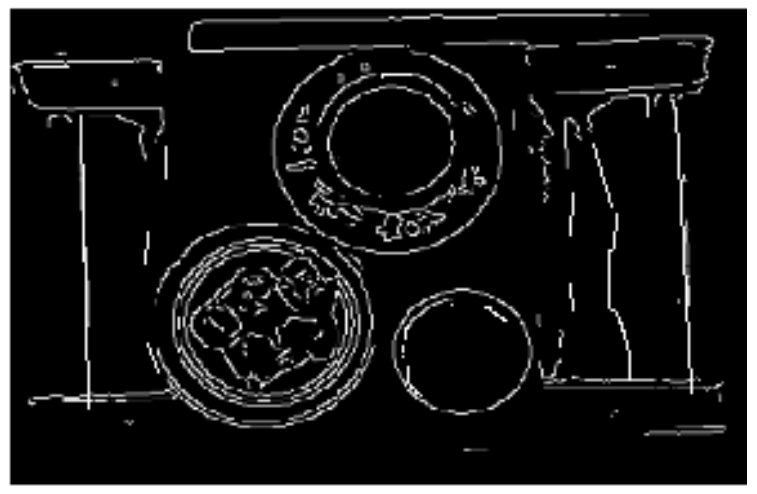

(b)

Fig. 2. Experimental results, (a)original image, (b) resultant contour.

There are many advantages of using rough contour detection tool and methodology. Firstly, the exhaust process is effective because the problem of space shrinks. Second, more convenient for the end user, which often turn into a rules of thumb.

The general framework of the MFCD model first computes the responses of neuron to the stimuli located within CRF. The three local cues of original image (luminance, luminance contrast, and orientation) [15] are extracted and corresponding inhibitory weights corresponding to center-surround difference are computed at each location. Then, a scale-guided combination approach is used to combine the three inhibitory weights, which is then modulated by the surround inhibition. In contrast to predictive methods, this particle filtering methodology is automatic process and contextually dependent and it treat semi local region. The final response calculating at each location is obtained by subtracting the surround inhibition from the CRF response. By learning their previous distributions, it forces the mathematical limitations which can reduce the efficiency of an algorithm.

\section{CONCLUSIONS}

Multi-scale particle filter is one of the suitable method to detect contours in images. This method is also suitable to detect contours of complex image. The set of pixels set is simulated on both scales, which normally embeds semi-local information. The presented model is combination of the frequent number-based method zones and contour presentation skills. This method is easy and also attractive because it has a compact set of linear controls. As an application to this approach, regionbased user interfaces are added in the context of our proportion-based contour detection. Improved results are more balanced design / contour cues in the region could eliminate complex object contours.

\section{REFERENCES}

[1] Widynski, N., \& Mignotte, M. (2014). A multiscale particle filter framework for contour detection. IEEE transactions on pattern analysis and machine intelligence, 36(10), 1922-1935.

[2] Ming, Y., Li, H., \& He, X. (2015). Winding Number Constrained Contour Detection. IEEE Transactions on Image Processing, 24(1), 68-79.

[3] Kurmi, Y., \& Chaurasia, V. (2015). An Image Fusion Approach based on Adaptive Fuzzy Logic Model with Local Level Processing. International Journal of Computer Applications, 124(1), 39-42.

[4] Arbelaez, P., Maire, M., Fowlkes, C., \& Malik, J. (2011). Contour detection and hierarchical image segmentation. IEEE transactions on pattern analysis and machine intelligence, 33(5), 898-916.

[5] Sun, J., Xu, Z., \& Shum, H. Y. (2011). Gradient profile prior and its applications in image super-

Devshri Swami, Bharti Chaurasia and Yashwant Kurmi, "A Multiscale Particle Filter and Multi-Feature Based Contour Detection," International Journal of Advanced Engineering and Management, Vol. 2, No. 6, pp. 127-130, 2017. https://doi.org/10.24999/IJOAEM/02060031 
resolution and enhancement. IEEE Transactions on Image Processing, 20(6), 1529-1542.

[6] Sharma, D., Kurmi, Y., \& Chaurasia, V. (2014). Formation of Super-Resolution Image: A Review. Int. Jour. of Emerging Tech. and Adv. Engg, 4(4), 218-221.

[7] Feroz, F., Hobson, M. P., \& Bridges, M. (2009). MultiNest: an efficient and robust Bayesian inference tool for cosmology and particle physics. Monthly Notices of the Royal Astronomical Society, 398(4), 1601-1614.

[8] Doucet, A., Briers, M., \& Sénécal, S. (2006). Efficient block sampling strategies for sequential Monte Carlo methods. Journal of Computational and Graphical Statistics, 15(3), 693-711.

[9] Yang, K. F., Li, C. Y., \& Li, Y. J. (2014). Multifeature-based surround inhibition improves contour detection in natural images. IEEE Transactions on Image Processing, 23(12), 50205032.

[10] Shan, C., Tan, T., \& Wei, Y. (2007). Real-time hand tracking using a mean shift embedded particle filter. Pattern recognition, 40(7), 1958-1970.

[11] Sun, X., Shang, K., Ming, D., Tian, J., \& Ma, J. (2015). A biologically-inspired framework for contour detection using superpixel-based candidates and hierarchical visual cues. Sensors, 15(10), 2665426674.

[12] Vinje, W. E., \& Gallant, J. L. (2000). Sparse coding and decorrelation in primary visual cortex during natural vision. Science, 287(5456), 1273-1276.
[13] Ghanbari, M., Majdi, M., \& Talouki, M. (2017). Video Inpainting Using a Contour-based Method in Presence of More than One Moving Objects. International Journal of Advanced Engineering and Management, 2(2), 37-44.

[14] Widynski, N., \& Mignotte, M. (2012). A particle filter framework for contour detection. Computer Vision-ECCV 2012, 780-793.

[15] Fowlkes, C. C., Martin, D. R., \& Malik, J. (2007). Local figure-ground cues are valid for natural images. Journal of Vision, 7(8), 2-2.

\section{Devshri Swami}

Dept. of Electronics \& Communication Engineering, Scope College of Engineering, Bhopal, India email: devshri.swami@gmail.com

\section{Bharti Chaurasia}

Dept. of Electronics \& Communication Engineering, Scope College of Engineering, Bhopal, India email: bharti.chourasia27@gmail.com

\section{Yashwant Kurmi}

Dept. of Electronics \& Communication Engineering, Maulana Azad National Institute of Technology Bhopal, India email: yashwantkurmi18@gmail.com 